International Conference Mathematical and Computational Biology 2011 International Journal of Modern Physics: Conference Series

Vol. 9 (2012) 537-542

(C) World Scientific Publishing Company

DOI: $10.1142 /$ S2010194512005636

\title{
ON SOME NEW PROPERTIES OF CATALAN NUMBERS
}

\author{
ISAMIDDIN S. RAKHIMOV and KAMEL A. MOHD ATAN \\ Department of Mathematics, Faculty of Science \& \\ Institute for Mathematical Research (INSPEM), \\ Universiti Putra Malaysia, \\ 43400, UPM, Serdang, Selangor Darul Ehsan, Malaysia \\ risamiddin@gmail.com
}

\begin{abstract}
It is well known that Catalan Numbers are used in Mathematical Biology as Enumerating RNA secondary structures for finite points. In this paper, we present some new properties of the Catalan Numbers.
\end{abstract}

Keywords: Nucleotide, RNA structure; secondary structure; Catalan numbers.

PACS numbers: 92D10, 05A10, 05A19.

\section{Introduction}

In combinatorial mathematics, the Catalan numbers form a sequence of natural numbers that occur in various counting problems, often involving recursively defined objects. They are named after the Belgian mathematician Eugène Charles Catalan (1814-1894), who discovered the connection to parenthesized expressions during his exploration of the Towers of Hanoi puzzle.

The $n$th Catalan number is given directly in terms of binomial coefficients by

$$
C_{n}=\frac{1}{n+1}\left(\begin{array}{c}
2 n \\
n
\end{array}\right)=\frac{(2 n) !}{(n+1) ! n !} \quad \text { for } n \geq 0 .
$$

The first Catalan numbers for $n=0,1,2,3, \ldots$ are

$1,1,2,5,14,42,132,429,1430,4862,16796,58786,208012,742900$,

2674440, 9694845, 35357670, 129644790, 477638700, 1767263190, 6564120420,

24466267020, $91482563640,343059613650,1289904147324,4861946401452, \ldots$

The Catalan sequence was first described in the 18th century by Leonhard Euler, who was interested in the number of different ways of dividing a polygon into triangles. 


\subsection{A few combinatorial applications}

The book Enumerative Combinatorics, Volume 2 by combinatorial mathematician Richard P. Stanley contains a set of exercises which describe 66 different sequences of sets with the property that the $n$-th set of each collection has the same number $C_{n}$ of objects. (See http://www-math.mit.edu/ rstan/ec/catalan.pdf)

Here are some examples

- Let a binary operation - on a set is given and let $x_{1} x_{2} \ldots x_{n}$ be a word. Then the number of parenthesizing of this word is given by the Catalan number $C_{n}$.

- Let $A_{n}$ be a regular $n$ polygon $(n \geq 3)$. Then $C_{n-2}$ is a number of possible triangulations of $A_{n}$.

- Nonnesting matchings on $2 n$, i.e., ways of connecting $2 n$ points in the plane lying on a horizontal line by $n$ arcs, each arc connecting two of the points and lying above the points, such that no arc is contained entirely below another.

- Ways of connecting $2 n$ points in the plane lying on a horizontal line by $n$ vertex-disjoint arcs, each arc connecting two of the points and lying above the points, such that the following condition holds: for every edge $e$ let $n(e)$ be the number of edges $e^{\prime}$ that nest $e$ (i.e., $e$ lies below $e^{\prime}$ ), and let $c(e)$ be the number of edges $e^{\prime}$ that begin to the left of $e$ and that cross $e$. Then $n(e)-c(e)=0$ or 1 .

- Ways of connecting any number of points in the plane lying on a horizontal line by nonintersecting arcs lying above the points, such that the total number of arcs and isolated points is $n-1$ and no isolated point lies below an arc.

- Ways of connecting $n$ points in the plane lying on a horizontal line by noncrossing arcs above the line such that if two arcs share an endpoint $p$, then $p$ is a left endpoint of both the arcs.

- Ways of connecting $n+1$ points in the plane lying on a horizontal line by noncrossing arcs above the line such that no arc connects adjacent points and the right endpoints of the arcs are all distinct.

- Ways of connecting $n+1$ points in the plane lying on a horizontal line by $n$ noncrossing arcs above the line such that the left endpoints of the arcs are distinct.

- Ways of connecting $2 n-2$ points labeled $1,2, \ldots, 2 n-2$ lying on a horizontal line by nonintersecting arcs above the line such that the left endpoint of each arc is odd and the right endpoint is even.

\subsection{Properties of Catalan numbers}

- $C_{0}=1$ and $C_{n+1}=\sum_{i=0}^{n} C_{i} C_{n-i}$ for $n \geq 0$.

- $C_{n}=\frac{1}{n+1} \sum_{i=0}^{n}\left(\begin{array}{c}n \\ i\end{array}\right)^{2}$. 
- $C_{0}=1$ and $C_{n}=\frac{2(2 n-1)}{n+1} C_{n-1}$.

\subsection{An application in mathematical biology}

RNA molecules are particularly fascinating since they represent both: genotypic legislative via their primary sequence and phenotypic executive via their functionality associated to $2 D$ or $3 D$-structures, respectively. Accordingly, it is believed that RNA may have been instrumental for early evolution-before proteins emerged. The primary sequence of an RNA molecule is the sequence of nucleotides $A, G, U$ and together with the Watson-Crick $(A-U, G-C)$ and $(U-G)$ base pairing rules specifying the pairs of nucleotides can potentially form bonds. Single stranded RNA molecules form helical structures whose bonds satisfy the above base pairing rules and which, in many cases, determine their function. For instance, RNA ribozymes are capable of catalytic activity, cleaving other RNA molecules. RNA secondary structure prediction is of polynomial complexity (Waterman, 1978) which is the result from the fact that in secondary structures no two bonds can cross.

Primary Structure. An RNA molecule is a sequence of nucleotides of four possible types, denoted by the letters $A, C, G$ and $U$, connected by a backbone and is called RNA Primary Structure.

Base Pairing. Two nucleotides that are connected via hydrogen bonds are called a base pair. In the Watson-Crick base pairing, $A$ always forms a base pair with $U$, as does $G$ with $C$. In the Wobble base pairing, $G$ forms a base pair with $U$.

Notation. An RNA sequence of length $n$ is assumed as a sequence of $n$ points $(1-2-\ldots-n)$, in which each point $i, 1<i<n$, is connected to $i-1$ and $i+1$. The notation $i . j$ stands for the nucleotide $i$ is paired with the nucleotide $j$ and $i<j$.

RNA Structure. An RNA structure is a set $S$ of base pairs $i . j$ with $1 \leq i<$ $j \leq n$, such that for any $i_{1} \cdot j_{1}, i_{2} \cdot j_{2} \in S: i_{1}=i_{2} \Longleftrightarrow j_{1}=j_{2}$.

Secondary Structure. The set $S$ is called secondary structure if for all $i_{1} \cdot j_{1}, i_{2} \cdot j_{2} \in S$ they are nested, i.e., $i_{1}<i_{2}<j_{2}<j_{1}$, or disjoint, i.e., $i_{1}<j_{1}<i_{2}<j_{2}$.

About three decades ago, Waterman et al. pioneered the concept of RNA secondary structures (Penner and Waterman; ${ }^{1}$ Waterman ${ }^{2},{ }^{3}$ Schmitt and Waterman; ${ }^{4}$ Howell et al. ${ }^{5}$ ). The key property of secondary structures is best understood, considering a structure as a diagram, which is obtained as follows: one draws the primary sequence of nucleotides horizontally and ignores all chemical bonds of its backbone. Then, one draws all bonds, i.e., nucleotide interactions satisfying the Watson-Crick base pairing rules (and $G-U$ pairs) as arcs in the upper half plane, effectively identifying structure with the set of all arcs. In this representation, RNA secondary structures have then following property: there exist no two $\operatorname{arcs}\left(i_{1}, j_{1}\right),\left(i_{2}, j_{2}\right)$, where $i_{1}<j_{1}$ and $i_{2}<j_{2}$ with the property $i_{1}<i_{2}<j_{1}<j_{2}$ and all arcs have at least length 2. Equivalently, there exist no two arcs that cross in the diagram representation of the structure, see Fig. 1. 

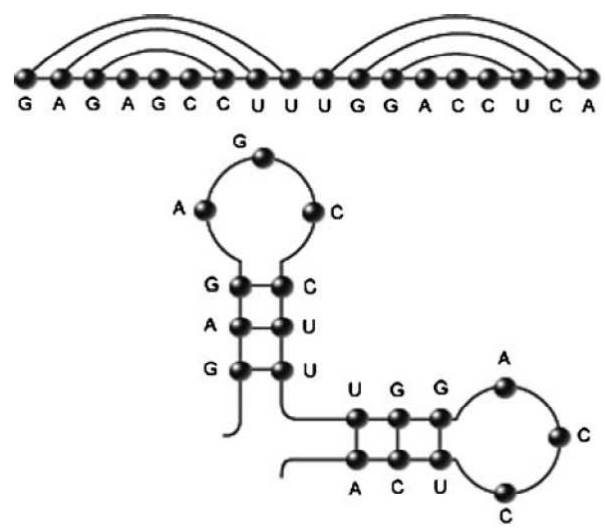

Fig. 1. RNA secondary structures. Diagram representation (top): the primary sequence, $G A G A G C C U-U U G G A C C U C A$, is drawn horizontally and its backbone bonds are ignored. All bonds are drawn in the upper half plane and secondary structures have the property that no two arcs intersect and all arcs have minimum length 2. Outer planar graph representation (bottom).

Basically, all combinatorial properties of secondary structures are derived from Waterman's basic recursion (Waterman, 1978)

Theorem 1.1. Let $C_{n}$ be the number of secondary structures for $n$ points. Then $C_{1}=C_{2}=1$, and for $n>2, C_{n}$ satisfies

$$
C_{n}=C_{n-1}+\sum_{k=1}^{n-1} C_{k} C_{n-k-1}
$$

where $C_{0}=1$.

\section{Results}

For the Catalan numbers $C_{k}, \quad k=1,2, \ldots$ the following identities hold true

- $\sum_{k=3}^{t-1}\left(\begin{array}{l}k-1 \\ k-2\end{array}\right) C_{t-k} C_{k-2}=\left(\begin{array}{c}t-3 \\ 1\end{array}\right) C_{t-2}$;

- $\sum_{k=3}^{t-1}\left(\begin{array}{l}k-1 \\ k-2\end{array}\right) C_{t-k} \sum_{l=3}^{k-1}\left(\begin{array}{l}l-1 \\ l-2\end{array}\right) C_{k-l} C_{l-2}$

$-\sum_{k=3}^{t-1}\left(\begin{array}{l}k-1 \\ k-3\end{array}\right) \sum_{i=k+2}^{t} C_{t+1-i} C_{i-1-k} C_{k-2}$

$=\left(\begin{array}{c}t-3 \\ 2\end{array}\right) C_{t-2}$

- $\sum_{k=3}^{t-1}\left(\begin{array}{c}k-1 \\ k-2\end{array}\right)\left(\begin{array}{c}k-3 \\ 2\end{array}\right) C_{t-k} C_{k-2}$ 


$$
\begin{aligned}
&-\sum_{k=3}^{t-1}\left(\begin{array}{c}
k-1 \\
k-3
\end{array}\right)\left(\begin{array}{c}
k-3 \\
1
\end{array}\right) \sum_{i_{1}=k+2}^{t} C_{t+1-i_{1}} C_{i_{1}-1-k} C_{k-2} \\
&+\sum_{k=3}^{t-1}\left(\begin{array}{c}
k-1 \\
k-4
\end{array}\right) \sum_{i_{2}=k+3}^{t} \sum_{i_{1}=k+3}^{i_{2}} C_{t+1-i_{2}} C_{i_{2}+1-i_{1}} C_{i_{1}-k-2} C_{k-2} \\
&=\left(\begin{array}{c}
t-3 \\
3
\end{array}\right) C_{t-2} ; \\
& \text { - } \sum_{k=3}^{t-1}\left(\begin{array}{c}
k-1 \\
k-2
\end{array}\right)\left(\begin{array}{c}
k-3 \\
3
\end{array}\right) C_{t-k} C_{k-2}
\end{aligned}
$$$$
-\sum_{k=3}^{t-1}\left(\begin{array}{c}
k-1 \\
k-3
\end{array}\right)\left(\begin{array}{c}
k-3 \\
2
\end{array}\right) \sum_{i_{1}=k+2}^{t} C_{t+1-i_{1}} C_{i_{1}-1-k} C_{k-2}
$$$$
+\sum_{k=3}^{t-1}\left(\begin{array}{l}
k-1 \\
k-4
\end{array}\right)\left(\begin{array}{c}
k-3 \\
1
\end{array}\right) \sum_{i_{2}=k+3}^{t} \sum_{i_{1}=k+3}^{i_{2}} C_{t+1-i_{2}} C_{i_{2}+1-i_{1}} C_{i_{1}-k-2} C_{k-2}
$$$$
-\sum_{k=3}^{t-1}\left(\begin{array}{l}
k-1 \\
k-5
\end{array}\right) \sum_{i_{3}=k+4}^{t} \sum_{i_{2}=k+4}^{i_{3}} \sum_{i_{1}=k+4}^{i_{2}} C_{t+1-i_{3}} C_{i_{3}+1-i_{2}} C_{i_{2}+1-i_{1}} C_{i_{1}-k-2} C_{k-2}
$$$$
=\left(\begin{array}{c}
t-3 \\
4
\end{array}\right) C_{t-2} \text {. }
$$

In general,

- $A_{k}\left(t, j_{1}, j_{2}, \ldots, j_{k-1}\right)=\sum_{j_{1}=3}^{t-1}\left(\begin{array}{c}j_{1}-1 \\ j_{1}-2\end{array}\right) C_{t-j_{1}} A_{k-1}\left(t, j_{1}, j_{2}, \ldots, j_{k-2}\right)$

$-\sum_{j_{1}=3}^{t-1}\left(\begin{array}{c}j_{1}-1 \\ j_{1}-3\end{array}\right) \sum_{i_{1}=j_{1}+2}^{t} C_{t+1-i_{1}} C_{i_{1}-1-j_{1}} A_{k-2}\left(t, j_{1}, j_{2}, \ldots, j_{k-3}\right)$

$+\sum_{j_{1}=3}^{t-1}\left(\begin{array}{c}j_{1}-1 \\ j_{1}-4\end{array}\right) \sum_{i_{2}=j_{1}+3}^{t} \sum_{i_{1}=j_{1}+3}^{i_{2}} C_{t+1-i_{2}} C_{i_{2}+1-i_{1}} C_{i_{1}-j_{1}-2} A_{k-3}\left(t, j_{1}, j_{2}, \ldots, j_{k-4}\right)$

$$
+\ldots+(-1)^{k} \sum_{j_{1}=3}^{t-1}\left(\begin{array}{l}
j_{1}-1 \\
j_{1}-k
\end{array}\right) \sum_{i_{k-2}=j_{1}+k-1}^{t} \sum_{i_{k-3}=j_{1}+k-1}^{i_{k-2}}
$$

. . $\sum_{i_{1}=j_{1}+(k-1)}^{i_{2}} C_{t+1-i_{k-2}} C_{i_{k-1}+1-i_{k-3}} \ldots C_{i_{2}+1-i_{1}} C_{i_{1}-j_{1}-2} A_{1}\left(j_{1}\right)$

$$
+(-1)^{k+1} \sum_{j_{1}=3}^{t-1}\left(\begin{array}{c}
j_{1}-1 \\
j_{1}-(k+1)
\end{array}\right) \sum_{i_{k-2}=j_{1}+k}^{t} \sum_{i_{k-3}=j_{1}+k}^{i_{k-2}}
$$$$
\sum_{i_{1}=j_{1}+k}^{i_{2}} C_{t+1-i_{k-2}} C_{i_{k-1}+1-i_{k-3}} \ldots C_{i_{2}+1-i_{1}} C_{i_{1}-j_{1}-2} C_{j_{1}-2}
$$

$=\left(\begin{array}{c}t-3 \\ k\end{array}\right) C_{t-2}$ 
The proofs of all the identities are immediate if replace the Catalan numbers by binomial coefficients and use some well known combinatorial identities.

\section{Acknowledgments}

The author acknowledges the Universiti Putra Malaysia (Malaysia) for support via grant 05-01-09-0733RU.

\section{References}

1. R. C. Penner and M. S. Waterman, Spaces of RNA secondary structures, Adv. Math. 101, 31-49 (1993).

2. M. S. Waterman, Secondary structure of single-stranded nucleic acids, Adv. Math. I (suppl.) 1, 167-212 (1978).

3. M. S. Waterman, Combinatorics of RNA hairpins and cloverleafs, Stud. Appl. Math. 60, 91-96 (1979).

4. W. R. Schmitt and M. S. Waterman, Linear trees and RNA secondary structure, Discret. Apl. Math. 51, 317-323 (1994).

5. J. A. Howell, T. F. Smith and M. S. Waterman, Computation of generating functions for biological molecules, SIAM J. Appl. Math. 39, 119-133 (1980). 